\title{
Clinical-Pathological Conference: Case 5
}

\author{
Craig B. Fowler $\cdot$ Nisha J. D’Silva
}

Received: 14 June 2010/Accepted: 10 July 2010/Published online: 30 July 2010

(C) US Government 2010

\section{Clinical Presentation}

A 20 month-old female was referred to the pediatric dentistry department for dental restorative procedures. Her medical history was remarkable for cerebral palsy and a congenital encephalocele. Oral examination revealed a relatively normal appearing dentition for her age, and her alveolar mucosa and periodontium also appeared within normal limits. All remaining intraoral mucosa was unremarkable with the exception of bilateral elongated nodules on the left and right lateral borders of her tongue. The nodules, which had been present since birth, were soft to palpation, slightly paler than the surrounding mucosa, and asymptomatic (Fig. 1). Review of her electronic medical record also indicated that she carried a diagnosis of Apert syndrome, although no further details were available.

\section{Differential Diagnosis}

Clinically, two, pale-colored, soft tissue exophytic masses were observed bilaterally on the antero-lateral tongue. Delayed tooth eruption was noted. The patient appeared to

C. B. Fowler $(\bowtie)$

Oral and Maxillofacial Pathology, 59 Dental Training Squadron/ SGDTM, 2200 Bergquist Drive Suite 1, Lackland AFB,

San Antonio, TX 78236-5300, USA

e-mail: craig.fowler@us.af.mil

\section{N. J. D'Silva}

Department of Periodontics and Oral Medicine, University of Michigan School of Dentistry, 1011 North University Avenue, Room G018, Ann Arbor, MI 48109-1078, USA

e-mail: njdsilva@umich.edu be a mouth breather with a trapezoidal shaped mouth. Given the patient's medical history of Apert syndrome, encephalocele and cerebral palsy, the differential diagnosis included swellings associated with accumulation of mucopolysaccharides, foreign material with bilateral scarring, ectopic neuroglial tissue and lingual hamartomas.

The clinical phenotype of Apert syndrome includes craniosynostosis, midface malformations with relative mandibular prognathism, a depressed nasal bridge and beaked nose, downslanting palpebral fissures, low set ears and syndactyly of the hands and feet [1]. The oral findings of Apert syndrome have been described by Kreiborg and Cohen [2]. These findings include lateral swelling of the palatine processes, a cleft uvula, tooth crowding, bulging/ thickened alveolar processes, and delayed dental eruption [2]. The palatine process swelling may be due to accumulation of mucopolysaccharides which sometimes leads to pseudoclefting of the palate [2]. The patients have delayed dental eruption. Accumulation of mucopolysaccharides has not been described at other intraoral sites, so this is an unlikely cause of the lingual swellings.

The possibility of foreign material with scarring of the tongue was considered but a surgical procedure that involved implantation of foreign material was not reported. Given the young age of the patient, it is unlikely that she was able to embed a foreign object in the tongue that went unnoticed long enough for healing to take place. Therefore, this possibility was ruled out.

There has been a case report of ectopic neuroglial tissue occurring in the tongue in a patient with encephalocele but this was an isolated case not associated with a syndrome [3]. Hence, it seemed unlikely to be the cause of the bilateral tongue swellings.

Lingual hamartoma is the favored diagnosis. These are the 3rd most common tongue lesions in children preceded by 


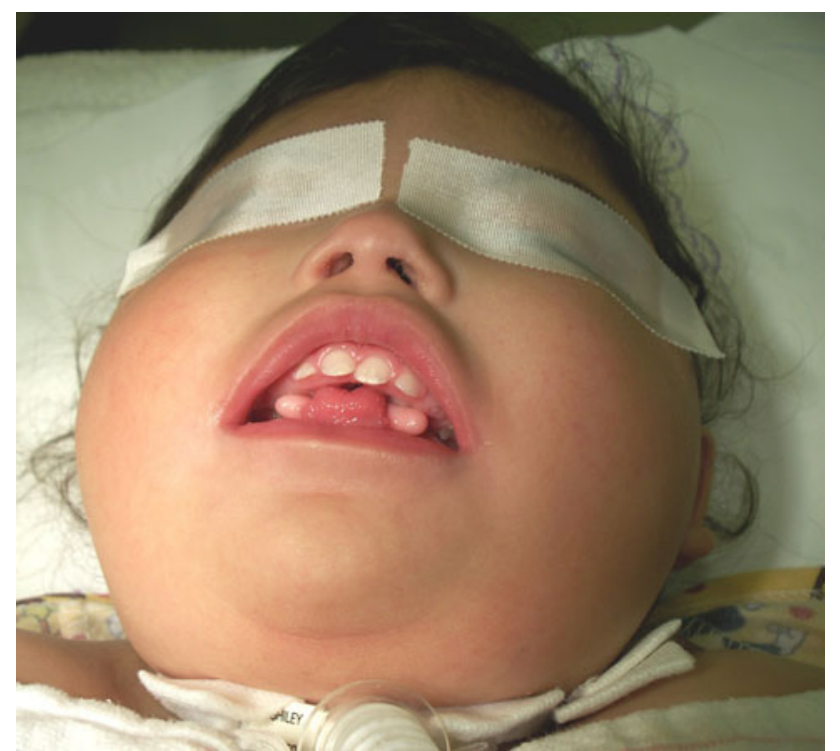

Fig. 1 Clinical photo showing bilateral tongue nodules

vascular/lymphatic malformations and mucus extravasation phenomenon (mucocele) [4]. In a review of 135 tongue lesions in children, 18 of these were lingual hamartomas [4]. They usually present as submucosal masses. Sixteen of the 18 cases presented on the dorsum of the tongue. They exhibit a female predilection and usually present at less than 2 years of age. Ten of the 18 cases consisted of smooth muscle and fat, but lesions that were primarily fat, or smooth muscle or neurovascular were also observed [4]. Lingual hamartomas usually occur as isolated cases [4, 5]. However, four of the 18 cases reported on the tongue of children were associated with oral-facial-digital syndrome [4].

There are several types of oral-facial-digital syndrome that have oral findings, facial anomalies and digital anomalies such as syndactyly and polydactyly in common [1]. The OFD Type I syndrome is inherited as a dominant $\mathrm{X}$-linked trait but most cases are sporadic [1]. Oral manifestations include hyperplastic frenula, midline cleft in the upper lip, clefts in the palate and fibrotic bands in the mucobuccal fold [1]. Cleft tongues with two to multiple lobes are seen in $75 \%$ of cases [1]. Hamartomas are observed on the ventral tongue in about $70 \%$ of cases [1]. Malposed or supernumerary teeth may also be observed [1]. In a recent study of 12 patients with OFD Type I, lingual hamartomas were observed in all patients [6]. There is some overlap in the clinical features of OFD and Apert syndrome including minor facial anomalies, cleft palate and digital anomalies. Given the clinical presentation of the swellings on the tongue, the history of Apert syndrome with the overlap in some clinical features with OFD Type I, the favored diagnosis was lingual hamartoma (x2) possibly in association with OFD Type I.

\section{Diagnosis and Discussion}

The patient was taken to the operating room to complete the restorative procedures, and the nodules were removed at the same visit under general anesthesia. The patient tolerated the procedures well and there has been no recurrence of the nodules.

Microscopic evaluation of the nodules revealed both to be composed primarily of mature adipose tissue. Lobules of minor salivary glands, skeletal muscle, and occasional smooth muscle were also noted (Figs. 2, 3, 4) A diagnosis of "fat predominant hamartoma" was rendered.

Review of the English literature back to 1950 revealed 58 reported cases of tongue hamartomas, including the largest review of 18 cases [4-9]. Four of the patients had

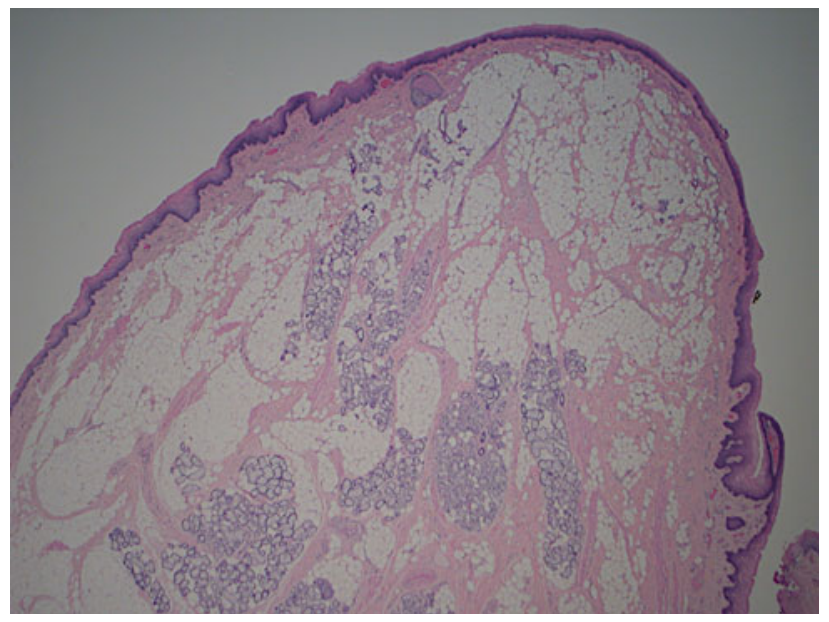

Fig. 2 Low-power photomicrograph of tongue nodule showing predominance of mature adipose tissue and salivary glands. (Original magnification $\times 20$ )

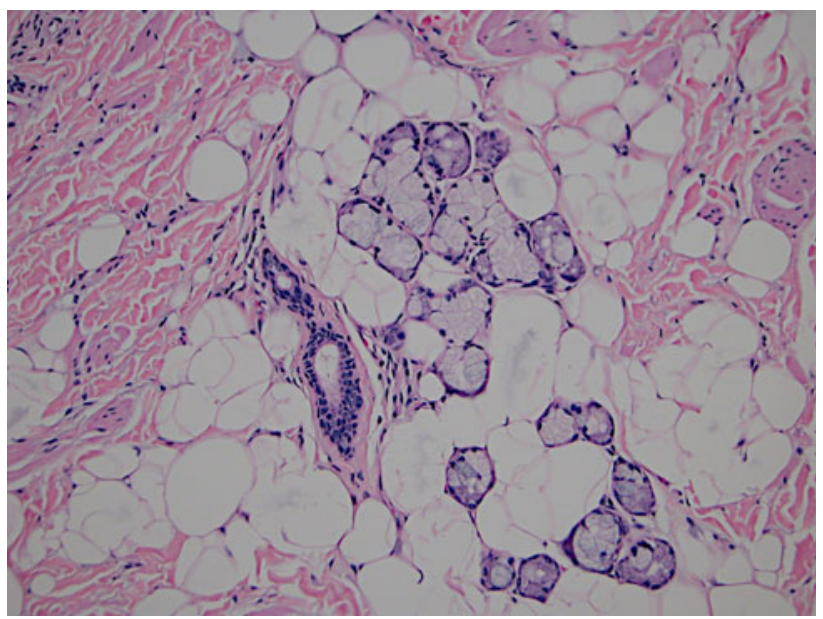

Fig. 3 Medium-power photomicrograph of tongue nodule showing minor salivary gland lobules and duct. (Original magnification $\times 100$ ) 


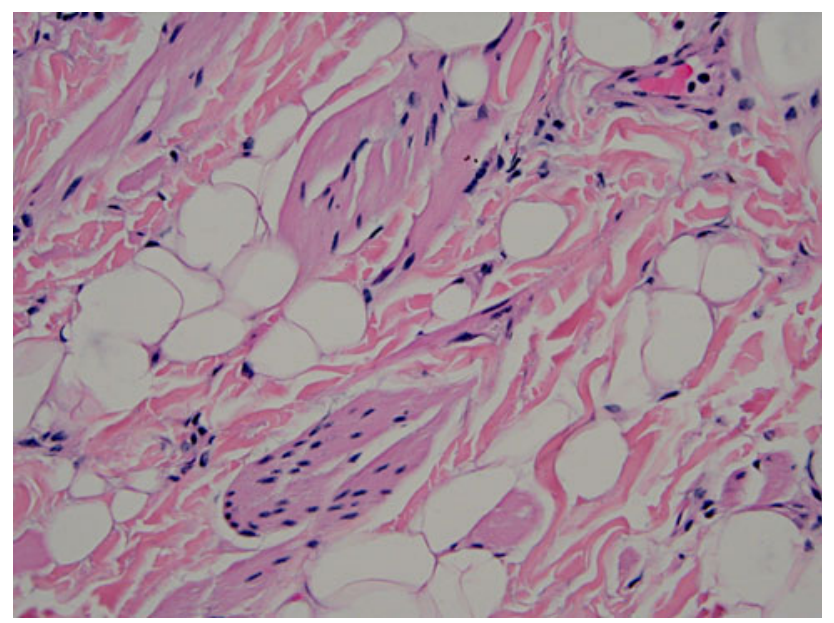

Fig. 4 High-power photomicrograph of tongue nodule showing smooth muscle bundles. (Original magnification $\times 200$ )

multiple lesions; therefore, a total of 68 lesions were found. In 12 cases, detailed information regarding age, gender, specific location on the tongue, and microscopic description of the hamartomas was lacking [6]. The remaining 46 cases involved 32 females and 14 males for a 2.3:1 maleto-female ratio. The age at diagnosis ranged from birth to 74 years. Twenty-eight of the cases were diagnosed at or before 1 year of age. Eight cases were not diagnosed until after the first decade; however, five of those eight were documented to have been present at birth. The majority of lesions $(N=23)$ were located at the base of tongue or middorsal tongue in the midline, 14 were located on the lateral border or lateral dorsal tongue, 10 at the anterior dorsal or tip, and five on the ventral surface. Four additional lesions were located on the dorsal tongue and tongue, respectively, without further specification regarding location.

Microscopic composition of the previously reported tongue hamartomas is detailed in Table 1 . Most of the lesions contained smooth muscle, salivary glands, fat, and/ or blood vessels. Nerves, skeletal muscle, sebaceous glands, and lymphoid tissue were also noted in some cases. Of interest is the fact two cases contained cartilage and bone, respectively, but were reported as "hamartomas". One could argue that these lesions would be more appropriately termed "choristomas" since neither bone nor cartilage normally occur in the tongue.

Most cases of tongue hamartomas are not associated with any systemic condition, but 18 cases in the literature were documented to occur in association with Oral-Facial Digital Syndrome (OFDS) [4, 6-8, 10], and one in association with Ectrodactyly-Ectodermal Dysplasia-Cleft Lip and Palate Syndrome (EECS) [9]. No cases of tongue hamartomas in Apert syndrome were documented. Because of this, the diagnosis of Apert syndrome in our patient was questioned and further information was sought regarding
Table 1 Microscopic composition of 56 tongue hamartomas (English literature)

\begin{tabular}{ll}
\hline Tissue type & Number of lesions \\
\hline Smooth muscle & 35 \\
Salivary glands & 25 \\
Fat & 22 \\
Blood vessels & 22 \\
Nerves & 10 \\
Skeletal muscle & 8 \\
Sebaceous glands & 3 \\
Lymphoid tissue & 2 \\
Cartilage & 1 \\
Bone & 1 \\
\hline
\end{tabular}
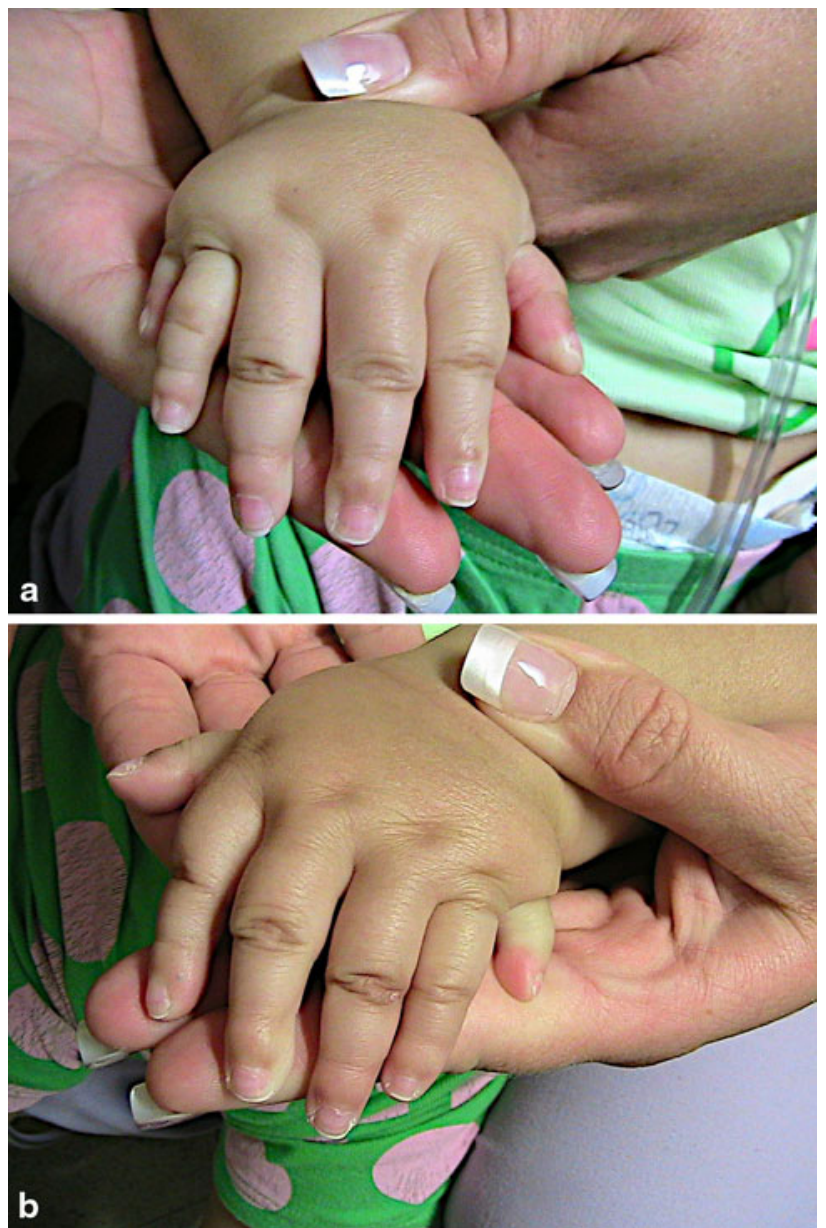

Fig. 5 a, b Clinical photo of hands showing post-axial polydactyly (hexadactyly), clinodactyly, and brachydactyly of thumbs, 5th and 6th fingers

the patient's hands and feet. Consultation with our pediatric neurologist revealed that the patient had hexadactyly of both hands and both feet, but no syndactyly was identified (Figs. 5, 6). 

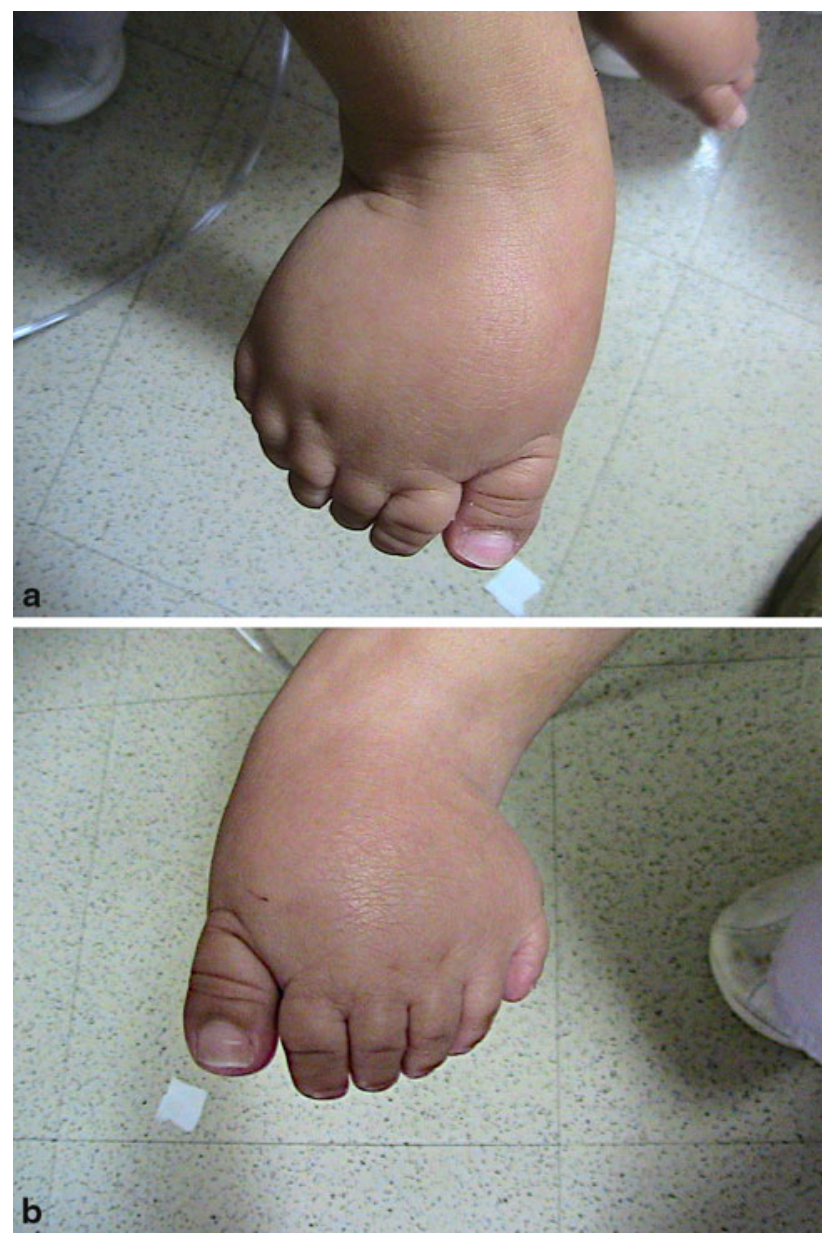

Fig. 6 a, b Clinical photo of feet showing post-axial polydactyly (hexadactyly), and brachydactyly

OFDS is a complex group of syndromes showing heterogeneity. Common to all are minor facial anomalies, oral findings including cleft or lobulated tongue, oral frenula, and/or cleft palate, and digital anomalies including brachydactyly, syndactyly, clinodactyly, and polydactyly [1]. To date, 11 types of OFDS have been reported as well as some miscellaneous or unclassifiable forms $[1,10]$. Tongue hamartomas have been reported in types I, II, III, IV, VI, VII, VIII, and IX [10]. According to our pediatric geneticist, our patient is thought to have a recessive form of OFDS, but is chromosomally normal on testing to date. Given the clinical findings and inheritance pattern in our patient, she appears to most closely conform to OFDS, type II (Mohr syndrome); however, she does not have a midline cleft of the upper lip as described in many patients with this form of OFDS. Therefore, she may represent a miscellaneous or unclassifiable form of OFDS.

Acknowledgments The authors wish to thank Col John Embry and Maj Brian Faux for providing the clinical images used in this paper.

\section{References}

1. Gorlin RJ, Cohen MM Jr, Hennekam RCM. Syndromes of the head and neck. 4th ed. Oxford: Oxford University Press; 2001. p 654-58, 832-44, 878-82.

2. Kreiborg S, Cohen MM Jr. The oral manifestations of Apert syndrome. J Craniofac Genet Dev Biol. 1992;12(1):41-8.

3. Plontke SK, Preyer S, Pressler H, et al. Glial lesion of the infratemporal fossa presenting as a soft tissue middle ear massrudimentary encephalocele or neural crest remnant? Int J Pediatr Otorhinolaryngol. 2000;56(2):141-7.

4. Kreiger PA, Ernst LM, Elden LM, et al. Hamartomatous tongue lesions in children. Am J Surg Pathol. 2007;31(8):1186-90.

5. Iida S, et al. Multiple leiomyomatous hamartoma in the oral cavity. J Oral Pathol Med. 2007;36(4):241-4.

6. Tagliani MM, Gomide MR, Carrara CF. Oral-facial-digital syndrome type 1: oral features in 12 patients submitted to clinical and radiographic examination. Cleft Palate Craniofac J. 2010;47(2): $162-6$.

7. Sakai N, Nakahita N, Yamazaki Y, et al. Oral-facial-digital syndrome type II (Mohr syndrome): clinical and genetic manifestations. J Craniofac Surg. 2002;13(2):321-6.

8. Ghossaini SN, Hadi U, Tawil A. Oral-facial-digital syndrome type II variant associated with congenital tongue lipoma. Oral Surg Oral Med Oral Pathol Oral Radiol Endod. 2002;94:324-7.

9. Hanna R, Argenyi ZB, Benda JA. Hamartoma of the tongue in an infant with a primary diagnosis of ectrodactyly-ectodermal dysplasia-cleft lip and palate syndrome. J Cutan Pathol. 1994;21: 173-8.

10. Fujiwara I, Kondo Y, Iinuma K. Oral-facial-digital syndrome with hypothalamic hamartoma, postaxial ray hypoplasia of the limbs, and vagino-cystic communication: a new variant? Am J Med Genet. 1999;83:77-81. 